Proc. 17th Winter Workshop on

Nuclear Dynamics (2001) 000-000

17th Winter Wokshop on Nuclear Dynamics

Park City, Utah, USA

March 10-17, 2001

\title{
ORLaND - Oak Ridge Laboratory for Neutrino Detectors
}

\author{
F. Plasil ${ }^{1}$ for the ORLaND Project and for the CoNDOR Collaboration \\ ${ }^{1}$ Physics Division, Oak Ridge National Laboratory \\ Oak Ridge, TN 37831-6372
}

\begin{abstract}
The proposed Oak Ridge Laboratory for Neutrino Detectors (ORLaND), to be located adjacent to the Spallation Neutron Source (SNS), is described. ORLaND will take advantage of the fact that the SNS will be the world's best intermediateenergy pulsed neutrino source in the world. A broad range of neutrino measurements is contemplated by means of a number of detectors, including the large CoNDOR detector. Specifics of neutrino oscillation investigations, and of the possible impact of certain neutrino measurements on our understanding of supernova explosions, are discussed.
\end{abstract}

Keywords: Neutrinos, Spallation

PACS: 14.60.Lm, 14.60.Pq, 25.30.-c, 95.85.Ry

\section{Introduction}

The Spallation Neutron Source (SNS), under construction at the Oak Ridge National Laboratory, will not only be the world's most intense pulsed neutron source, but it will also be the most intense, pulsed, intermediate-energy neutrino source in the world. This provides the neutrino research community with a unique opportunity to build a laboratory in which a number of state-of-the-art neutrino measurements could be performed. This can be accomplished at a minimum cost, because the SNS accelerator complex has been funded and is already under construction. It will generate neutrinos whether or not their production is taken advantage of. In this paper we first present the status and design characteristics of the SNS. We then address neutrino production at the SNS and the properties of the produced neutrinos (flavor, intensity, spectra, etc.). We next turn to a description of the proposed Oak Ridge Laboratory for Neutrino Detectors (ORLaND) [1] and to the physics that can be addressed, with an emphasis on possible neutrino oscillation investigations. We will conclude with a summary and with our evaluation of future prospects.

ISBN 9630000000

(C) 2001 EP Systema, Debrecen 


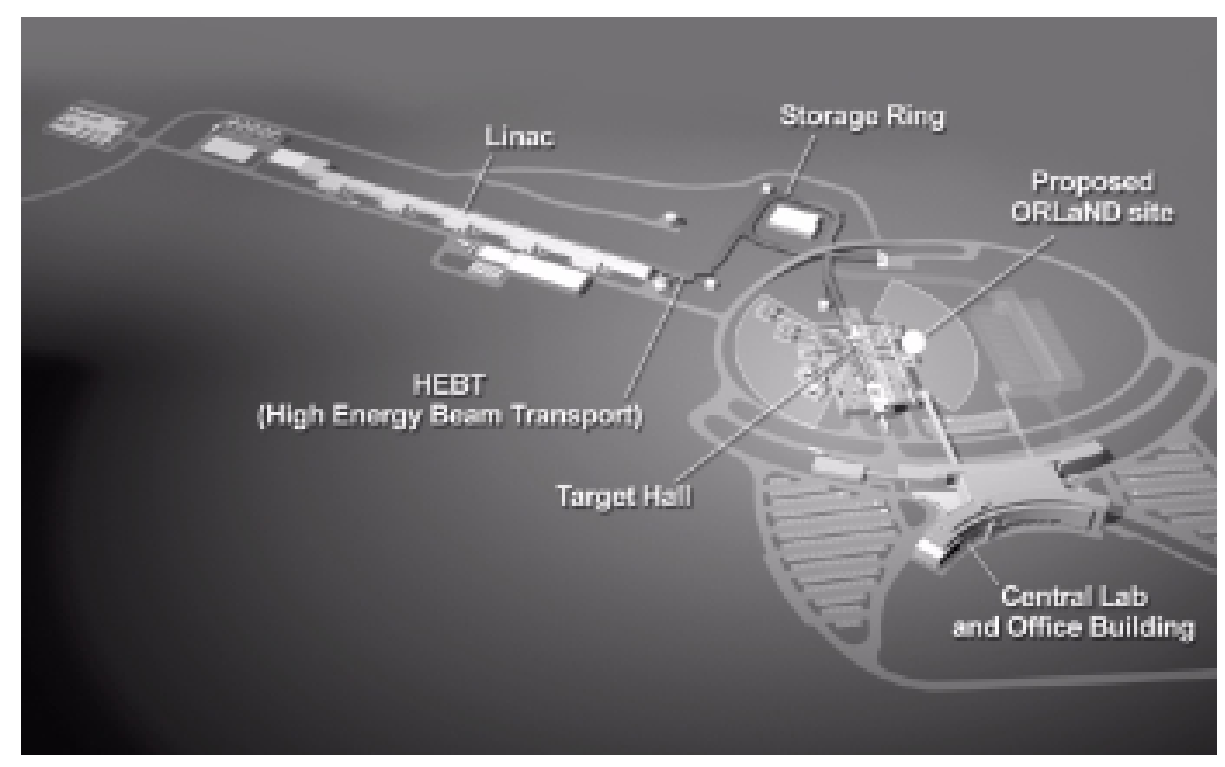

Fig. 1. Artist's concept of the Neutron Spallation Source (SNS) under construction at the Oak Ridge National Laboratory. The proposed site for ORLaND is indicated.

\section{The Spallation Neutron Source at ORNL}

This facility is the flagship enterprise of the condensed matter, materials, and applied neutron scattering communities. Construction has been initiated, and the project is scheduled to be completed in 2006. The neutron beams are designed to have an intensity that will be ten times that of any other pulsed neutron source. A $1-\mathrm{GeV}, 2 \mathrm{MW}$ proton LINAC will feed an accumulator ring, which will produce pulses at the rate of $60 \mathrm{~Hz}$, having a FWHM of less than $400 \mathrm{nsec}$ per pulse, and delivering $2 \times 10^{14}$ protons per pulse onto a mercury target. The facility overview is shown in Fig. 1. The proton beam size is kept deliberately large in order to dissipate the large amount of energy contained in each beam pulse. The size of the beam incident on the target is $7 \times 21 \mathrm{~cm}$. The liquid mercury target dimensions are $10 \times 40 \times 65 \mathrm{~cm}$. Currently, two ambient water moderators are envisaged, along with two liquid hydrogen cryogenic moderators. There are provisions for 24 neutron beam lines, but, at this time, the funds available are sufficient to implement only three of them.

The SNS project received full requested funding in FY 2001 (\$278M). The total cost of the project is estimated to be $\$ 1.4$ billion. Site excavation has been completed, with the moving of $1.3 \mathrm{M}$ cubic yards of earth. All preliminary designs of buildings and accelerator components and of most initial instrumentation units have been completed. There are teams in place to design and develop 12 instruments to serve the community. 


\section{Neutrinos Produced at the SNS}

A byproduct of the operation of the SNS is the production of $9.4 \times 10^{14}$ neutrinos/second in $60 \mathrm{~Hz}$ pulses. As was stated above, this constitutes the most intense, pulsed, intermediateenergy neutrino source in the world. A great opportunity would be missed if we did not take advantage of this source of neutrinos. The pulsed nature of the neutrino source would drastically reduce the backgrounds from cosmic rays. In fact, the effect of the cosmic-ray background at the SNS, due to the pulsed nature of the source, would be equivalent to that of a standard cosmic-ray background found at about $2.3 \mathrm{~km}$ of water-depth equivalent! Another desirable feature of the SNS neutrino source is that it is dominated by decay at rest due to the thick, high- $\mathrm{Z}$ mercury production target. Consequently, the energy and time spectra of the neutrinos are well known and can be calculated in advance. These spectra are shown in Fig. 2 and Fig. 4, respectively, and their method of production is shown in Fig. 3, which illustrates one of the remarkable selective features of neutrino production from the incidence of protons on a heavy target. As is seen in Fig. 3, positive and negative pions are produced with similar yields $(0.068 /$ collision for positive pions and $0.049 /$ collision for negative pions.) However, the decay chain for negative pions is dominated by the capture of both the negative pions and of the subsequently produced negative muons, resulting in low yields (0.000016/collision) of $\overline{\mathrm{v}}_{e}$. In contrast, positive pions lead to copious production of $v_{\mu}, \bar{v}_{\mu}$, and $v_{e}$. Thus, the production of $\bar{v}_{e}$ is very much lower than the production of the other types of neutrinos, resulting in a $\bar{v}_{e}$ to $\bar{v}_{\mu}$ ratio of about $2.4 \times 10^{-4}$. This is essential for one of the most important experiments contemplated at ORLaND - the transition of $\overline{\mathrm{v}}_{\mu}$ to $\overline{\mathrm{v}}_{e}$, claimed to have been observed earlier [2].

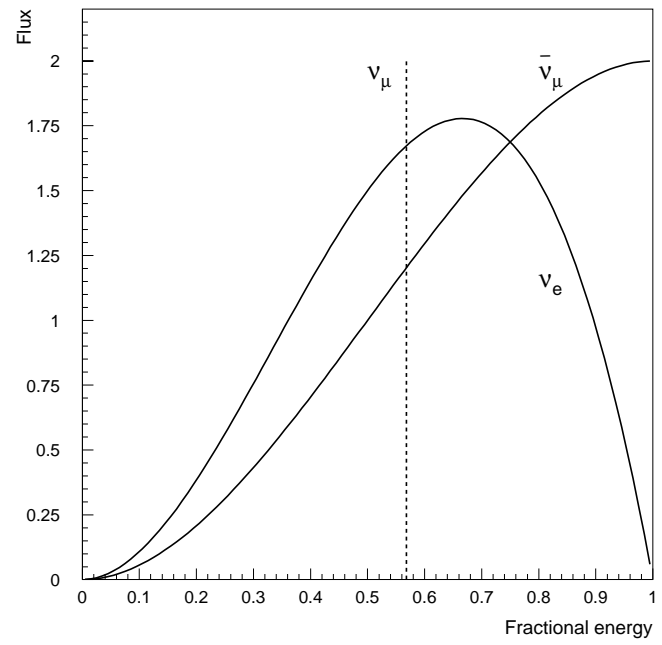

Fig. 2. Energy spectra of three neutrino species produced from the decay of $\pi^{+}$and $\mu^{+}$.

The neutrino spectra shown in Fig. 2 cover the intermediate-energy range up to about $55 \mathrm{MeV}$. This is the same energy range that is of interest in studies of the role of neutrinos 


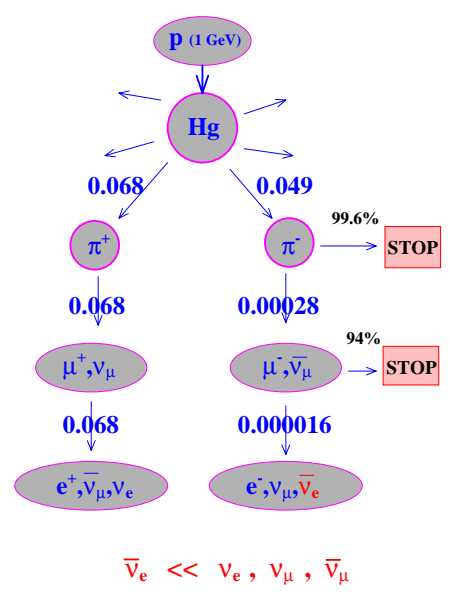

Fig. 3. Schematic of the production mechanism of different species of neutrinos at the SNS. Note the very low yield of $\bar{v}_{e}$ relative to the other neutrino species resulting from the large capture probability of both the negative pions and the negative muons.

in supernova explosions, providing us with an opportunity to perform experiments directly relating to these cataclysmic stellar events. Note that this energy range is not accessible either via neutrino production from reactors which results in neutrino energies below 10 $\mathrm{MeV}$, nor from traditional accelerator-based neutrino production in which higher energies dominate. The monoenergetic $v_{\mu}$ line in Fig. 2 is due to the decay at rest of $\pi^{+}$. Simulated time spectra of neutrinos produced at the SNS are shown in Fig. 4. These spectra illustrate the advantages of a pulsed source. For example, if one wishes to substantially reduce the background in the appearance of $\bar{v}_{e}$ neutrinos which may be produced from transitions of $\bar{v}_{\mu}$ neutrinos, it is possible to make measurements during the time intervals between SNS pulses, thus eliminating the time period which includes the high initial time peak in the irreducible background production of $\bar{v}_{e}$ from the decay of the few negative muons that have not been captured.

\section{The Proposed ORLaND Facility}

The proposed ORLaND site is indicated in Fig. 1. ORLaND [1] would consist of a concrete "bunker" large enough to accommodate one very large ( $2 \mathrm{k}$-ton) detector, and five to six smaller special-purpose detectors. The proposed bunker location is at $90 \mathrm{deg}$. to the incident proton beam direction on the north side of the target hall and as close to the target as is practical. A schematic drawing of the bunker and of the large detector inside it is given in Fig. 5. The large detector is located off-center within the bunker, leaving space for the smaller detectors to be located on the various platforms shown in the figure. The facility 

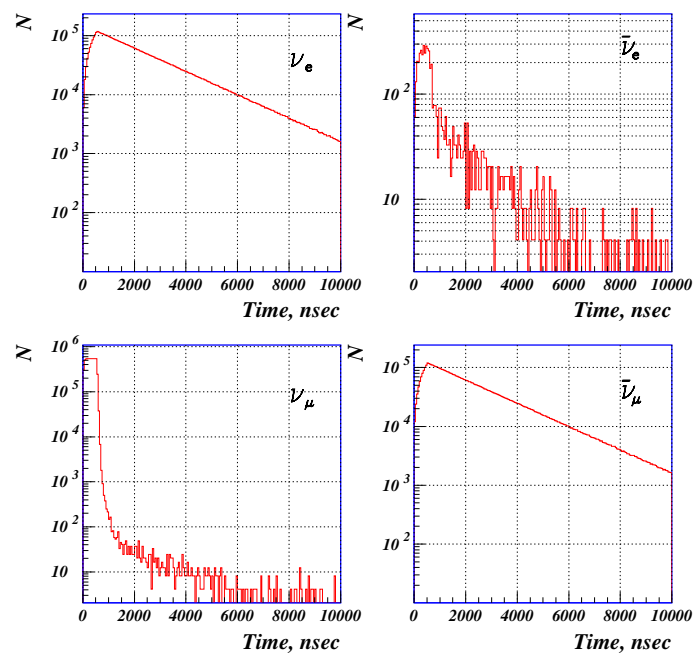

Fig. 4. Expected time distributions of four species of neutrinos produced at the SNS.

has a 30-m water equivalent overburden to reduce the background from cosmic rays. In the large detector, this background will be further reduced via an active veto shield. The approximate dimensions of the large detector are $14 \mathrm{~m}$ diameter and $14 \mathrm{~m}$ high. The bunker will be accessible via a separate dedicated tunnel with a modest incline, allowing easy access for personnel, equipment, and materials. Construction techniques have been identified which will allow the bunker to be built even after the completion of the target hall. It is anticipated that the large detector will be welded in situ.

The projected cost of the ORLaND facility, including the large detector and one or two smaller detectors, is expected to be about $\$ 65 \mathrm{M}$. Funding for the bunker is being sought from the Department of Energy, while funding the detectors, including the large detector, will be requested from both the NSF and the DOE. The ORLaND Project is centered in the Physics Division of the Oak Ridge National Laboratory and is being proposed in collaboration with the Oak Ridge Associated Universities. Several collaborations are in the process of forming with the purpose of proposing the construction of a number of specific detectors. The collaboration promoting the large detector and consisting of most of the original proponents of ORLaND has recently taken the name of CoNDOR (Comprehensive Neutrino Detector at Oak Ridge). It consists of members from about 25 institutions. Frank Avignone is the CoNDOR Spokesperson.

The proposed CoNDOR detector will consist of a large cylindrical steel vessel capable of containing about $2 \mathrm{k}$-tons of liquid, with a built-in veto system and a passive absorber between the veto layer and the inner wall of the tank. The readout system under consideration would consist of about 5000 13-inch photomultiplier tubes resulting in a spatial resolution of about $15 \mathrm{~cm}$. This level of accuracy is important for event vertex determination (which is critical for background rejection) and also for the accurate determination of the fiducial volume of the detector. These are both key ingredients that will allow us 


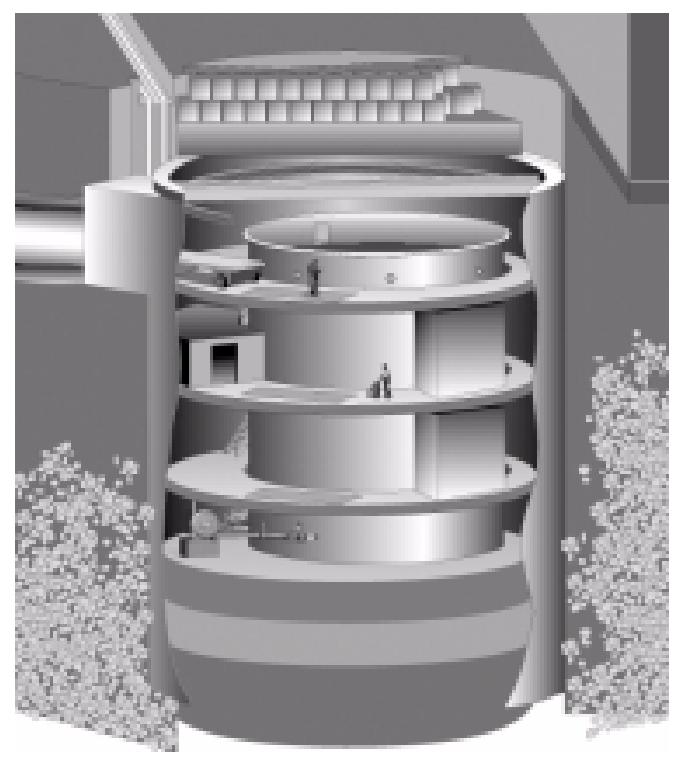

Fig. 5. Artist's rendition of the proposed ORLaND facility, including the large CoNDOR tank, located off-center inside the ORLaND bunker.

to make measurements at the required level of precision. It is contemplated that the detector will initially be filled with mineral oil containing a low concentration of scintillator so that both scintillator and Cherenkov light can be detected. Other options include the subsequent replacement of the mineral oil with water and/or the addition of an internal optically transparent smaller vessel containing heavy water for the possible measurement of neutrino-deuterium interactions.

\section{Neutrino Science at ORLaND}

Due to limitation of time and space, we will only list here the various areas of neutrino science that can be addressed by ORLaND. Detailed descriptions can be found in an ORNL document entitled "Scientific Opportunities at ORLaND", dated September 21, 2000 [3]. We have already mentioned in this paper the potential impacts in the areas of neutrino oscillations and supernova explosions, and we will expand on these two topics below. In addition, investigations in the following fields could also be pursued at ORLaND: role of neutrinos in hydrogen-burning in the core of the sun leading to a better understanding and to a possible refinement of the Standard Solar Model (SSM); hidden strangeness in nucleons via studies of neutrino-nucleus quasi-elastic scattering; precision measurement of the Weinberg angle which is very important for the interpretation of the Standard Model in the context of a complete renormalizeable theory; search for the effects of a possible mag- 
netic moment of the muon neutrino; neutral- and charged-current measurements on carbon relating to such issues as the Gamow-Teller quenching in neutrino-nuclear reactions; etc.

The original primary motivation of the small group that gathered in Oak Ridge in September 1996 to propose a neutrino facility associated with the SNS was the study of neutrino oscillations. This remains one of the main goals of CoNDOR. The existence of spontaneous transitions of neutrinos of one flavor to another flavor implies that neutrinos have a mass. This, in turn, has implications of major importance on our understanding of elementary particles and forces, and on certain aspects of cosmology and of astrophysics. Currently, there are three indications that neutrinos do, indeed, "oscillate" and, hence, have mass. Two of these rely on the disappearance of expected neutrino flux. (The solar neutrino puzzle, in which the flux of observed electron neutrinos from the sun, in a number of experiments, is consistently lower than the predictions of the SSM, and the Super-Kamiokande result [4] in which there appears to be a deficit in the muon neutrinos expected from cosmic rays impinging on earth's atmosphere.) Only one investigation, the LSND experiment at Los Alamos [2], claims to have evidence for the appearance of a neutrino flavor $\left(\bar{v}_{e}\right)$ that must have resulted from the transition of another neutrino flavor $\left(\bar{v}_{\mu}\right)$. Our initial motivation was to verify the LSND results by making a similar measurement, but with much increased sensitivity. A similar "confirmation" experiment, MiniBooNE, is being constructed at the Fermi National Accelerator Laboratory. It is probable that MiniBooNE will obtain the "confirmation" results before CoNDOR will have a chance to do so. However, regardless of the MiniBooNE result (confirming or negating the LSND results), the data that CoNDOR could provide would be invaluable. If the LSND result is verified, CoNDOR could explore the parameter space (in the usual $\sin ^{2} 2 \theta$ vs. $\delta \mathrm{m}^{2}$ ) with great precision, thus establishing the values of these important parameters. If , on the other hand, the LSND result is not confirmed, CoNDOR will be able to double-check this conclusion and also address areas of parameter space of interest to Supernova studies, and to studies relating to our understanding of Big Bang nucleo-synthesis.

The parameter space of interest to the Supernova and Big Bang studies is depicted in Fig. 6. Indicated are the regions of sensitivity of the KARMEN2 experiment (the first attempt, with negative results, to verify the LSND claim), of the MiniBooNE experiment, and of CoNDOR. The hatched regions show the areas of parameter space that are of interest to the cosmology and astrophysics communities. (SN stands for Supernova and BBN for Big Bang nucleo-synthesis. Note the "cross-hatched" region, of interest to both areas of research.) It is clear that CoNDOR will be able to address the regions of interest in a much more effective way than MiniBooNE will be able to.

CoNDOR measurements would make several contributions to our understanding of Supernova explosions. In the current view of these cataclysmic events, the gravitational collapse of the iron core of a massive star is followed by a rebounding shock wave which stalls due to energy losses resulting from nuclear dissociation and from neutrino emission. It is believed that an intense flux of neutrinos subsequently emitted from the center of the collapsed star re-energizes the explosion, and that these neutrinos interact with the outer layer resulting in a form of "neutrino nucleosynthesis" in which some naturally occurring isotopes are produced for which no other production mechanism is known. Given the important role of neutrinos in theories of supernova processes, measurements of neutrino- 


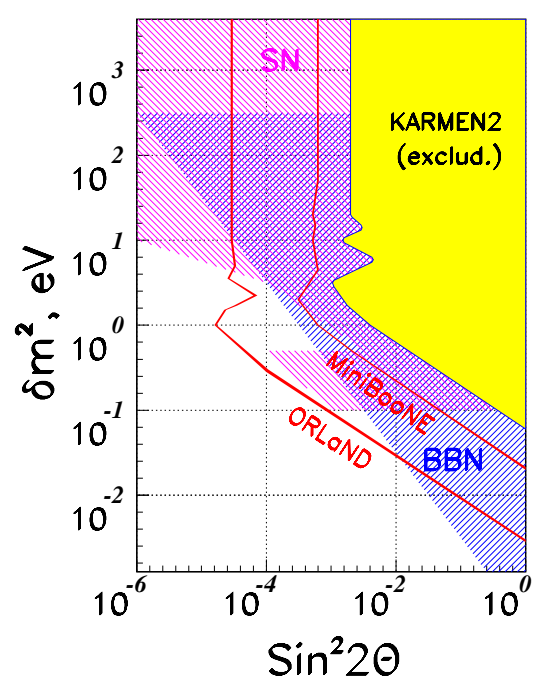

Fig. 6. CoNDOR sensitivity relating to investigations of possible neutrino oscillations. Note the hatched areas, beyond the reach of current and anticipated experiments, of interest to communities studying supernova explosions ( $\mathrm{SN}$ ) and Big Bang nucleosynthesis (BBN).

nucleus cross sections would provide verification of current theories and important input to theoretical modeling of supernova events. Such measurements would address areas of supernova dynamics, supernova nucleo-synthesis, and supernova neutrino detection here on earth. To have a major impact on these fields, the measurements need to be made at a level of accuracy below 5\%. This can be achieved with the proposed ORLaND detectors for a number of relevant isotopes spanning a broad mass range. Currently only the neutrinocarbon cross section is known at this level of accuracy.

\section{Summary and Conclusion}

The Oak Ridge Spallation Neutron Source will provide the neutrino research community with a unique and cost-effective opportunity to establish a neutrino detector center where a broad range of neutrino measurements in the intermediate-energy range could be made. These measurements would have important consequences for our understanding of many aspects of fundamental physics, cosmology, and astrophysics. The ORLaND concept has been under development for more than four years, and significant design and engineering work has been accomplished. Proposals to DOE and the NSF are in preparation. At a recent meeting of the nuclear physics community at which a new five-year plan for this research field was considered, the proposed ORLaND facility was identified as one of five important initiatives having a projected cost under $\$ 100 \mathrm{M}$. We are optimistic regarding its ultimate implementation. 


\section{ORLaND Project and the CoNDOR Collaboration}

B. Anderson ${ }^{h}$, F. T. Avignone ${ }^{y}$, T. C. Awes ${ }^{m}$, C. Baktash ${ }^{m}$, J. B. Ball ${ }^{m}$, D. W. Bardayan ${ }^{m}$, J. R. Beene ${ }^{m}$, S. C. Berridge ${ }^{z}$, F. E. Bertrand ${ }^{m}$, Ed Bilpuch ${ }^{e}$, J. C. Blackmon ${ }^{m}$, R. N. Boyd ${ }^{n}$, C. L. Britton ${ }^{m}$, W. M. Bugg ${ }^{z}$, R. L. Burman ${ }^{i, u}$, J. Busenitz ${ }^{r}$, H. K. Carter ${ }^{l}$, L. Chatterjee ${ }^{c}$, T. V. Cianciolo ${ }^{m}$, H. O. $\mathrm{Cohn}^{z}$, M. Danilov ${ }^{f}$, D. J. Dean ${ }^{m}$, L. DeBraeckeleer ${ }^{e}$, P. Degtiarenko ${ }^{g}$, G. W. Dodson ${ }^{m}$, Y. V. Efremenko ${ }^{m, z}$, M. A. Elaasar ${ }^{p}$, A. R. Fazely ${ }^{o}$, T. A. Gabriel ${ }^{m}$, A. Galindo-Uribarri ${ }^{m}$, C. R. Gould ${ }^{w}$, Z. D. Greenwood ${ }^{k}$, V. Gudkov ${ }^{y}$,

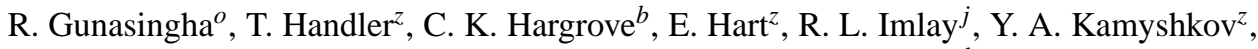
H. J. Karwowski ${ }^{v}$, D. D. Koetke ${ }^{a a}$, K. Kubodera ${ }^{y}$, K. Lande ${ }^{x}$, C. Lane ${ }^{d}$, R. W. Manweiler ${ }^{a a}$, W. J. Metcalf ${ }^{j}$, A. Mezzacappa ${ }^{m, z}$, G. B. Mills ${ }^{I}$, L. W. Mo ${ }^{b b}$, A. Murphy ${ }^{n}$, T. Nunamaker ${ }^{b b}$, S. Nussinov ${ }^{q}$, A. Piepke $r$, F. Plasil ${ }^{m}$, J. J. Reidy ${ }^{t}$, C. Rosenfeld ${ }^{y}$, D. Smith ${ }^{c c}$, I. Stancu ${ }^{r}$, S. Stanislaus ${ }^{a a}$, R. I. Steinberg ${ }^{d}$, M. R. Strayer ${ }^{m}$, R. C. Svoboda ${ }^{j}$, R. Tashakkori ${ }^{m}$, W. Tornow ${ }^{e}$, G. J. VanDalen ${ }^{s}$, J. Wolf ${ }^{r}$, G. R. Young ${ }^{m}$, O. Zeldovich ${ }^{f}$, W.-M. Zhang ${ }^{h}$

${ }^{a}$ Appalachian State University

${ }^{b}$ Carleton University

${ }^{c}$ Cumberland University

${ }^{d}$ Drexel University

${ }^{e}$ Duke University

${ }^{f}$ Institute for Theoretical \& Experimental Physics, Moscow

${ }^{g}$ Thomas Jefferson National Accelerator Laboratory

${ }^{h}$ Kent State University

${ }^{i}$ Los Alamos National Laboratory

${ }^{j}$ Louisiana State University

${ }^{k}$ Louisiana Tech

${ }^{l}$ Oak Ridge Associated Universities

${ }^{m}$ Oak Ridge National Laboratory

${ }^{n}$ Ohio State University

${ }^{o}$ Southern University at Baton Rouge

${ }^{p}$ Southern University at New Orleans

${ }^{q}$ Tel Aviv University

${ }^{r}$ University of Alabama

${ }^{s}$ University of California, Riverside

${ }^{t}$ University of Mississippi

${ }^{u}$ University of New Mexico

${ }^{v}$ University of North Carolina

${ }^{w}$ North Carolina State University

${ }^{x}$ University of Pennsylvania

${ }^{y}$ University of South Carolina

${ }^{z}$ University of Tennessee

${ }^{a}$ Valparaiso University

${ }^{b b}$ Virginia Tech

${ }^{c c}$ Embry-Riddle University 


\section{Acknowledgement(s)}

This research was sponsored by the Division of Nuclear Physics, U. S. Department of Energy under contract DE-AC05-00OR22725 managed by UT-Battelle, LLC. The help of Althea Tate, Yuri Efremenko, and Susan Jacques with the preparation of the manuscript is gratefully acknowledged.

\section{References}

1. "ORLaND”, internal ORNL and ORAU document, draft of a proposal, dated March 1999.

2. C. Athanassopoulus et al., Phys. Rev. Lett. 81 (1998) 1774.

3. "Scientific Opportunities at ORLaND", internal ORNL document, draft, dated September 21, 2000.

4. Y. Fukada et al., Phys. Lett. B433 (1998) 9. 